\title{
Dopamine Dependency of Oscillations between Subthalamic Nucleus and Pallidum in Parkinson's Disease
}

\author{
Peter Brown,, ${ }^{1}$ Antonio Oliviero, ${ }^{2}$ Paolo Mazzone, ${ }^{3}$ Angelo Insola, ${ }^{3}$ Pietro Tonali, ${ }^{2}$ and Vincenzo Di Lazzaro ${ }^{2}$ \\ ${ }^{1}$ Medical Research Council Human Movement and Balance Unit, Institute of Neurology, London WCIN 3BG, United \\ Kingdom, 2Institute of Neurology, Università Cattolica, 00168 Rome, Italy, and ${ }^{3} O$ perative Unit of Functional and \\ Stereotactic Neurosurgery, Centro Traumatologico Ortopedico, "A. Alesini" Hospital, 00145 Rome, Italy
}

The extent of synchronization within and between the nuclei of the basal ganglia is unknown in Parkinson's disease. The question is an important one because synchronization will increase postsynaptic efficacy at subsequent projection targets. We simultaneously recorded local potentials (LPS) from the globus pallidus interna (GPi) and subthalamic nucleus (STN) in four awake patients after neurosurgery for Parkinson's disease. Nuclei from both sides were recorded in two patients so that a total of six ipsilateral GPi-STN LP recordings were made. Without medication, the power within and the coherence between the GPi and STN was dominated by activity with a frequency
$<30 \mathrm{~Hz}$. Treatment with the dopamine precursor levodopa reduced the low-frequency activity and resulted in a new peak at $\sim 70 \mathrm{~Hz}$. This was evident in the power spectrum from STN and GPi and in the coherence between these nuclei. The phase relationship between the nuclei varied in a complex manner according to frequency band and the presence of exogenous dopaminergic stimulation. Synchronization of activity does occur between pallidum and STN, and its pattern is critically dependent on the level of dopaminergic activity.

Key words: globus pallidus interna; subthalamic nucleus; coherence; synchronization; Parkinson's disease; dopamine
Studies in 1-methyl-4-phenyl-1,2,3,6-tetrahydropyridine (MPTP)-treated primates and in patients with Parkinson's disease have found an increase in firing rate and a tendency toward bursting in the neurons of the globus pallidus interna (GPi) and subthalamic nucleus (STN) (Filion and Tremblay, 1991; Bergman et al., 1994; Sterio et al., 1994; Hutchison et al., 1997a, 1998; Merello et al., 1999). These changes are likely to influence the projection targets of the basal ganglia in the thalamus and brainstem, although not as much as if the postsynaptic efficacy of neuronal activity was increased through the synchronization of discharges emanating from these nuclei. There is some evidence for the synchronization of neuronal discharges within the GPi of MPTP-treated primates (Nini et al., 1995), but, to date, there is no evidence for significant synchronization in patients with Parkinson's disease.

Here we look for synchronization within and between the human GPi and ipsilateral STN in the presence and relative absence of dopaminergic stimulation, by recording from the basal ganglia in patients undergoing functional neurosurgery for severe Parkinson's disease. Patients were recorded after withdrawal and reinstitution of treatment with the dopamine precursor levodopa, which elevates levels of dopamine and its metabolites in the parkinsonian brain, without significant change in noradrenaline or serotonin (Scatton et al., 1983). To avoid surgery-related time constraints, we recorded local potentials (LPs) postoperatively from the different contacts of macroelectrodes rather than the action potentials of individual neurons using intraoperative mi-

Received July 11, 2000; revised Oct. 19, 2000; accepted Nov. 13, 2000.

This work was supported by the Medical Research Council. We thank J. C. Rothwell for his assistance in recording one of the patients and D. H. Halliday and D. Buckwell for computer programs.

Correspondence should be addressed to Dr. P. Brown, Medical Research Council Human Movement and Balance Unit, Institute of Neurology, Queen Square, London WCIN 3BG, UK. E-mail: p.brown@ion.ucl.ac.uk.

Copyright (C) 2001 Society for Neuroscience 0270-6474/01/211033-06\$15.00/0 croelectrodes. The use of bipolar contacts increased the likelihood that only local potentials were recorded because activity generated near one contact in a contact pair is more likely to give a deflection than activity generated at a distance and picked up by both contacts. We further confirmed the local generation of recorded potentials by demonstrating reversal in the polarity of the cumulant density estimates between consecutive bipolar pairs of contacts.

The LP is the product of synchronous activity in a population of neurons. In the cerebral cortex, the timing of neuronal discharge is closely related to fluctuations in the local field potential (LFP) (Creutzfeldt et al., 1966; Frost, 1968), and, by analogy, we considered fluctuations in the LP within GPi and STN to be a surrogate marker of the synchronization of neuronal discharge in these nuclei. The validity of this marker was checked by looking for coherence between GPi and STN, because the presence of the latter would suggest that LFP oscillations in GPi were locked to postsynaptic effects in STN and visa versa.

\section{MATERIALS AND METHODS}

Patients and surgery. All four patients (mean age of 59 years; range of 49-64 years; two females; mean duration of disease, 17 years; range of 9-27 years) participated with informed consent. Their mean United Parkinson's Disease Rating Scale (UPDRS) motor scores were 66 (range of 48-80) and 20 (range of 11-38) off and on medication, respectively. Patients took a mean daily dosage of $770 \mathrm{mg}$ of levodopa (range of $400-1500 \mathrm{mg}$ ) and a single dose of $200 \mathrm{mg}$ during the recording session. UPDRS scores on and off treatment and the efficacy of stimulation at different macroelectrode contacts were assessed independently of the experiment and blind to any of the results.

The operative procedure and beneficial clinical effects of stimulation have been described previously (Siegfried and Lippitz, 1994; Limousin et al., 1995; Starr et al., 1998). Macroelectrodes were inserted after GPi and STN had been identified by nontelemetric ventriculography and localized using microelectrode recording and microstimulation while the subject was awake. The coordinates at the tip of contact 0 were $19-24 \mathrm{~mm}$ from the midline of the patient, $2 \mathrm{~mm}$ in front of the midcommissural point, 
and $6 \mathrm{~mm}$ below the anterior commissure (AC)-posterior commissure (PC) line for Gpi, and $12 \mathrm{~mm}$ from the midline, $0 \mathrm{~mm}$ from the midcommissural point, and 4-5 mm below the AC-PC line for STN. Macroelectrode position was confirmed postoperatively using magnetic resonance imaging (MRI). The macroelectrodes in the pallidum and STN were models 3387 and 3389 (Medtronic Neurological Division, Minneapolis, $\mathrm{MN})$ with four platinum-iridium cylindrical surfaces $(1.27 \mathrm{~mm}$ diameter and $1.5 \mathrm{~mm}$ length) and center-to-center separations of 3 and $2 \mathrm{~mm}$, respectively. Contact 0 was the most caudal, and contact 3 was the most rostral. It was estimated that contacts $0-2$ of the pallidal electrode were potentially in GPi, whereas only contacts 0 and/or 1 of the STN electrode were in the STN. However, the exact position of the macroelectrode contacts cannot be determined antemortem. Postoperative stimulation of the macroelectrodes at clinically effective frequencies and intensities led to neither visual or somaesthetic responses nor muscle activation.

Recordings. Subjects were supine and were recorded at rest and while they performed isometric contraction of the wrist extensors, so as to maintain the wrist dorsiflexed by $\sim 30^{\circ}$ from the primary position. The forearm was supported in pronation, and wrist extension was unconstrained. During each task, LPs from the GPi and STN macroelectrodes were recorded simultaneously with contralateral wrist extensor EMG. EMG was picked up with bipolar 9-mm-diameter $\mathrm{Ag}-\mathrm{AgCl}$ electrodes. The degree of EMG activity was monitored on-line with verbal feedback being given so that activity was matched on and off medication. Deep brain activity was recorded from the adjacent four contacts of each macroelectrode $(0-1,1-2$, and 2-3). EMG was bandpass filtered at $10-300 \mathrm{~Hz}$ and amplified $(1000 \times)$. LPs and EEG were filtered at $1-300 \mathrm{~Hz}$ and amplified $(100-500,000 \times)$. Signals were sampled at $1 \mathrm{kHz}$ and recorded and monitored on-line using a customwritten program.

Analysis. A fast Fourier transform was performed on nonoverlapping sections of equal length (Halliday et al., 1995). Results were averaged across sections, and the autospectra, cross-spectra, and from this coherence were determined over $0-200 \mathrm{~Hz}$. A total of 1027 segments (mean of 178 per pair of nuclei from each subject) were averaged for each of the four conditions (rest and tonic voluntary contraction on and off levodopa). The segment length used was 1024 points, giving a frequency resolution of $0.98 \mathrm{~Hz}$. The variances of autospectra were stabilized using a logarithmic $(\log 10)$ transform. The variance of the coherence was normalized by transforming the square root of the coherence (a complex valued function termed coherency) at each frequency using the Fisher transform. This results in values of constant variance for each record given by $1 / 2 L$, where $L$ is the number of segment lengths used to calculate the coherence. A $\chi^{2}$ test was then used to test the hypothesis of equal coherence values in the original records at each frequency (Amjad et al., 1997). As multiple comparisons were performed, the confidence limits (CL) for autospectra, coherence, and $\chi^{2}$ spectra were calculated after a Bonferroni correction. Phase was only analyzed over those frequencies showing significant coherence between STN and GPi. The constant time lag between the two signals was calculated from the slope of the phase estimate after a line had been fitted by linear regression. The time lag was only calculated from the gradient if the number of contiguous data points included in the segment was equal to or more than eight, and a linear relationship accounted for $>80 \%$ of the variance. There often appeared to be more than one component of differing slope. The limits of individual components were defined by the turning points of the best-fit second- or third-order polynomial fitted to all contiguous plotted points. The polynomials accounted for $>80 \%$ of the variance and had the same or more than eight data points per model order. The results of phase analysis are useful in suggesting which nucleus is active first over a particular frequency band. However, $95 \% \mathrm{CL}$ were generally large so that inferences about the precise temporal differences between structures could not be made, especially because bipolar electrodes may degrade phase information (Mima and Hallett, 1999). It should also not be assumed that the lags and leads reported here only reflect conduction delays.

The cumulant density, equivalent to the cross-correlation between signals, was calculated from the inverse Fourier transform of the crossspectrum. Reversals in the polarity of the cumulant density estimate between consecutive bipolar pairs of contacts were used to determine the source of oscillatory activity. Only peaks in the cumulant density that were above the Bonferroni corrected 95\% CL were assessed.

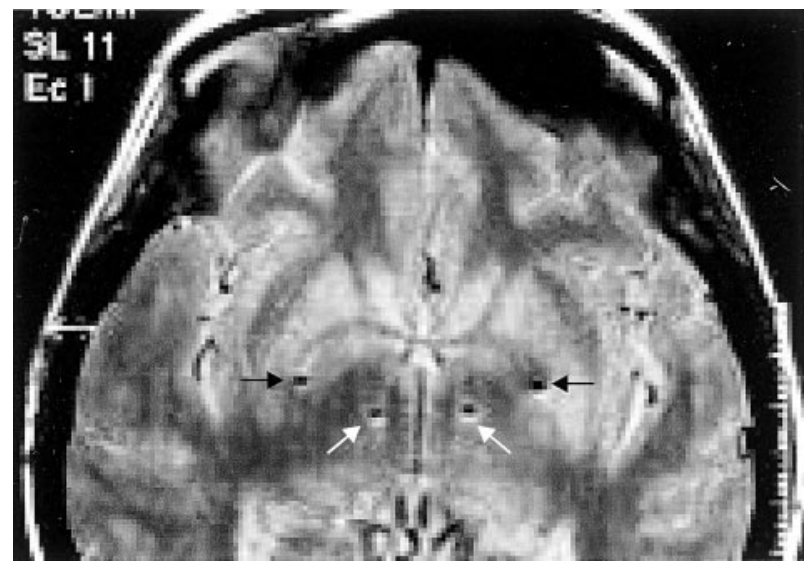

Figure 1. T1-weighted magnetic resonance axial view in a patient with Parkinson's disease after implantation of the left then right pallidum and STN. The sites of the deepest contacts $(0)$ are shown and lie in STN (white arrows) and GPi (black arrows), bilaterally. The scale to the right is in centimeters.

\section{RESULTS}

\section{GPi and STN LPs}

Four patients with idiopathic Parkinson's disease were studied 3-8 d after the simultaneous surgical implantation of macroelectrodes in the basal ganglia, in the period between implantation and subsequent subcutaneous rerouting to an internal stimulator (Fig. 1). Two patients were recorded twice as both sides were implanted over the course of the study. This resulted in LPs being sampled from a total of six GPi and six STN. Each patient was recorded at rest and during tonic voluntary contraction of the extensor muscles of the contralateral forearm after the overnight withdrawal of their antiparkinsonian medication and again $1.5 \mathrm{hr}$ after ingestion of levodopa. Figure 2 shows representative examples of the raw LP signals picked up in GPi and STN and variations in their frequency content over time. Off treatment, the records from both nuclei are dominated by oscillatory activity with a frequency below $30 \mathrm{~Hz}$ (Fig. $2 a$ ). These oscillations are reduced after the ingestion of levodopa, when a sharply tuned band of activity appears in STN at $\sim 70 \mathrm{~Hz}$ (Fig. $2 b$ ). Similar features were seen in all subjects. After treatment, two of the patients also showed a smaller peak in STN at $\sim 140 \mathrm{~Hz}$, a harmonic of the major activity at $70 \mathrm{~Hz}$ (Fig. 2c). One patient became drowsy during recordings made after levodopa, whereupon the $70 \mathrm{~Hz}$ oscillations became greatly diminished (Fig. 2d).

\section{STN-GPi coherence off levodopa}

Figures 3 and 5 illustrate the power, coherence, and phase spectra at rest and during tonic extension of the contralateral wrist in the different treatment states for activities recorded from macroelectrode contacts STN12 and GPi12, pooled across all subjects. These contacts were chosen because they included at least one contact from the pair yielding the best clinical improvement upon high-frequency stimulation in each patient and also demonstrated the highest coherence with one another. At rest, off antiparkinsonian medication, the coherence between LPs recorded at STN12 and GPi12 demonstrated two peaks (Fig. 3B). The first was relatively small and centered at $\sim 6 \mathrm{~Hz}$. STN led GPi at this frequency as shown by the net negative phase slope in Figure $3 E$. The second peak was much larger and centered at $\sim 20 \mathrm{~Hz}$. Up to $25 \%$ of the activity at this frequency was synchronized between GPi and STN. GPi led STN over this $\beta$ band, as shown by the net 


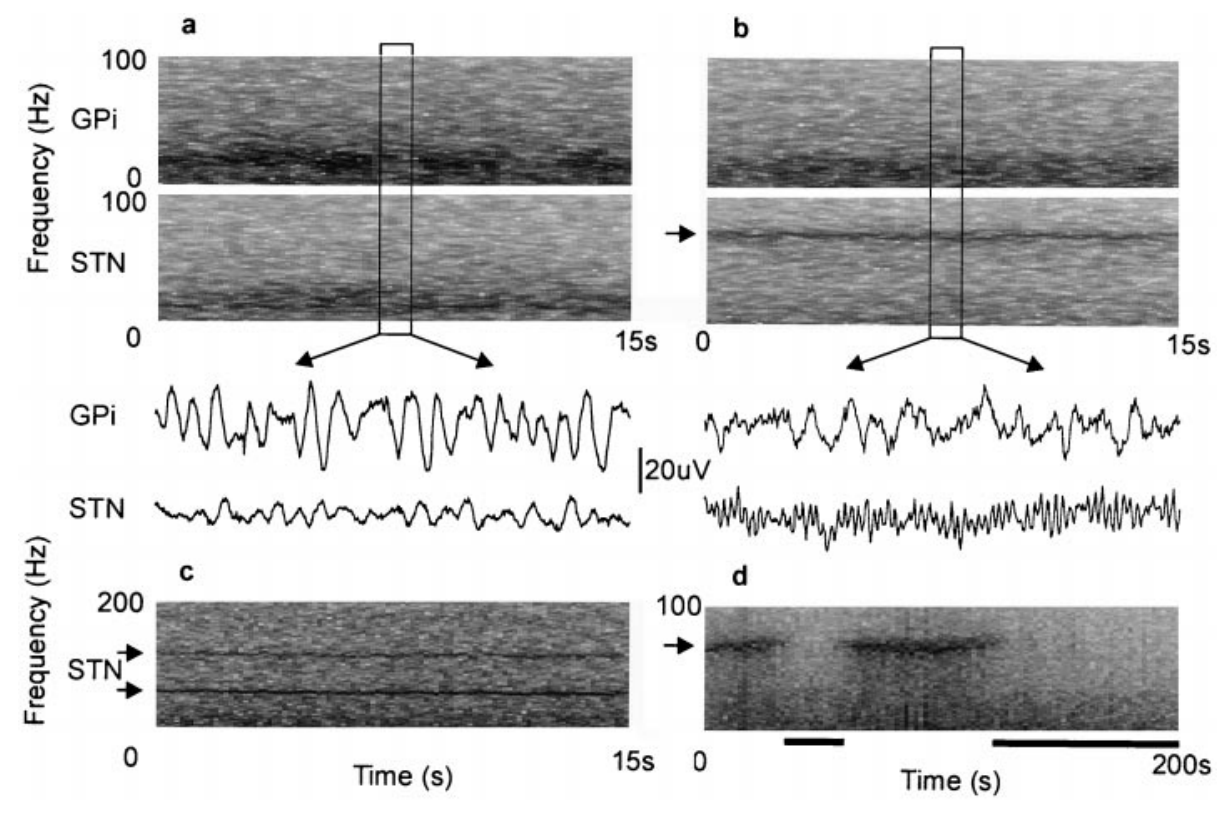

A

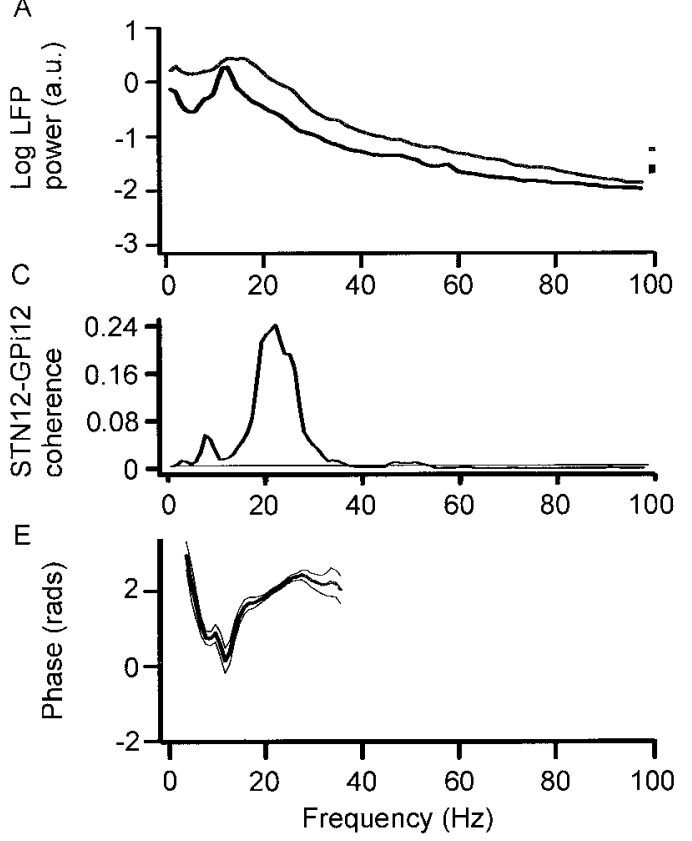

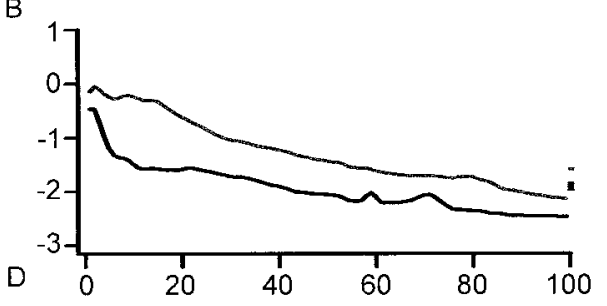

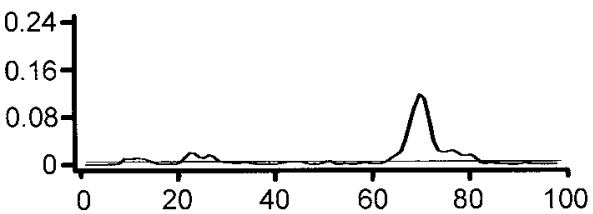

$F$

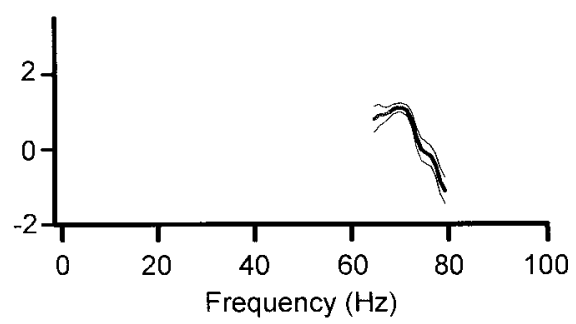

Figure 2. Raw LP signals picked up in GPi12 and STN12/01 at rest and variations in their frequency content over time. $a$, Off treatment, the records from both nuclei are dominated by activity with a frequency below $30 \mathrm{~Hz}$. $b$, Same patient after the ingestion of levodopa. Lowfrequency activity is reduced, and a sharply tuned band of activity appears in STN at $\sim 70 \mathrm{~Hz}$ (arrow). In both $a$ and $b$, a 1 sec segment of corresponding LP is shown under the frequency sonograms. $c$, Another patient recorded on treatment, showing activity in STN at $\sim 70$ and 140 $\mathrm{Hz}$ (arrows). $d$, A patient who fell drowsy during treatment with levodopa. The peak in activity at $\sim 70 \mathrm{~Hz}$ (arrow) is present when alert but not drowsy (denoted by black bars and defined as eyes closed and low-voltage slow activity and $\alpha$ dropout in EEG).

Figure 3. Autospectra of LP power $(A, B)$, coherence spectra between STN12 and GPi12 $(C, D)$, and respective phase spectra $(E, F)$ after withdrawal $(A, C, E)$ or reinstitution $(B, D, F)$ of levodopa. Data pooled from all records at rest in four patients with Parkinson's disease (over 6 experimental sessions). Off medication, there is coherence between STN12 and GPi12 at $\sim 6$ and $20 \mathrm{~Hz}$. Regression analysis of phase suggested that STN led GPi by $50 \pm 19$ msec from 3.9 to $11.7 \mathrm{~Hz}\left(r^{2}=0.853 ; p=\right.$ $0.0004)$, whereas GPi led STN by $20 \pm 5$ msec from 11.7 to $27.3 \mathrm{~Hz}\left(r^{2}=0.851 ; p<\right.$ 0.0001 ). It should be noted that the $95 \%$ confidence limits for these and ensuing temporal differences were broad, although they never encompassed zero. The lowfrequency activity is reduced on treatment when a peak appears at $\sim 70 \mathrm{~Hz}$ in the autospectrum of STN12 $(B)$ and coherence spectrum $(D)$. STN led GPi by $31 \pm 6 \mathrm{msec}$ $\left(r^{2}=0.928 ; p<0.0001\right)$. In this and Figure 5 , phase is shown in black (rather than gray) when it met criteria for measurement as defined in Materials and Methods, bin size is $0.98 \mathrm{~Hz}$, and vertical bars and thin lines are $95 \%$ CL in power spectra and in coherence and phase spectra, respectively.

positive slope in Figure $3 E$. Cumulant density estimates showed polarity reversal at contact 1 in GPi and STN (Fig. 4A), suggesting that oscillations arose at this contact and were not volume conducted from elsewhere. In the case of $\mathrm{GPi}$, which is big enough to accommodate several macroelectrode contacts, this result implies some functional somatotopy within the nucleus and is in accord with other evidence suggesting somatotopy in the human GPi (Bejjani et al., 1997; Krack et al., 1998).

The picture was similar during tonic contractions made off levodopa (Fig. 5C), except that coherence between STN12 and GPi12 was not as high (Fig. $6 C$, confirmed by $\chi^{2}$ test) and phase was indeterminate (Fig. $5 E$ ). Cumulant density estimates again showed polarity reversal at contact 1 in GPi and STN (data not shown).

\section{STN-GPi coherence after levodopa}

The picture was very different after levodopa. The latter was effective in improving parkinsonism (see Materials and Methods). Both at rest and during tonic activity power in the STN12 and GPi12, LPs dropped at frequencies under $30 \mathrm{~Hz}$ and a new peak appeared in the LPs at $\sim 70 \mathrm{~Hz}$ (Figs. $3 B, 5 B$ ). This was paralleled by a striking reduction in coherence between STN12 and GPi12 LPs at $\sim 20 \mathrm{~Hz}$ and by an increase in coherence at $\sim 70$ $\mathrm{Hz}$ (Figs. 3B, 5B). There was no significant coherence between STN12 and GPi12 above $90 \mathrm{~Hz}$ in the pooled or individual data. The differences between coherence spectra on and off levodopa were confirmed by a $\chi^{2}$ test on the pooled data (Fig. $6 A, B$ ). Thus, the synchronization of activities in GPi and STN shifted to much 
A

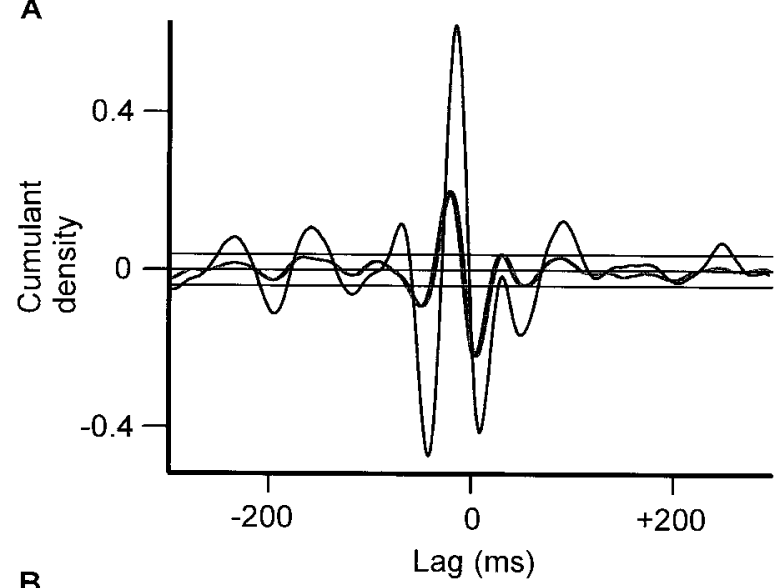

B

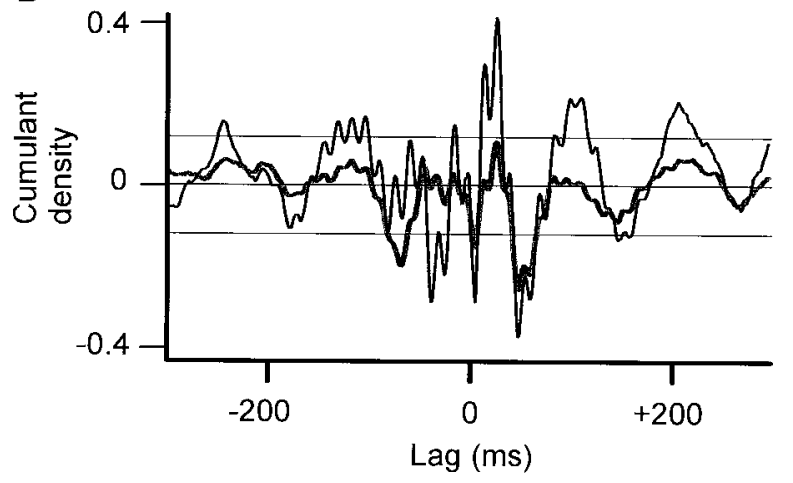

Figure 4. Cumulant density estimates after withdrawal $(A)$ and reinstitution of levodopa $(B)$ of levodopa recorded at rest. Black and thick gray lines are calculated from STN12-GPi12 and STN01-GPi12, respectively. STN01GPi12 has been inverted. The close superimposition of waves indicates polarity reversal around contact 1 in STN for both the slow activity in $A$ and fast $(70 \mathrm{~Hz})$ activity evident in $B$. The horizontal lines are the $95 \% \mathrm{CL}$.

higher frequencies after treatment with levodopa. STN tended to phase lead GPi over these higher frequencies, as shown by the net negative gradients in Figures $3 F$ and $5 F$. After treatment, cumulant density estimates showed polarity reversal of the $70 \mathrm{~Hz}$ activity at contact 1 in GPi and STN, suggesting that the fast oscillations arose within these nuclei (Fig. 4B), although during tonic wrist extension, there was an additional slow wave that did not phase reverse and probably represented volume conduction from a more distant source (data not shown). The coherence between STN12 and GPi12 LPs at $\sim 70 \mathrm{~Hz}$ after levodopa tended to be greater at rest than during tonic extension of the wrist (Fig. $6 D$, see $\chi^{2}$ test). Note that the small peak in coherence at $\sim 6 \mathrm{~Hz}$ at rest was greater off medication than after levodopa (Fig. 6A) and greater off medication but at rest than during tonic wrist extension (Fig. $6 C$ ), in keeping with a tremor-related phenomenon.

\section{DISCUSSION}

Our results show a marked change in the nature of oscillatory activity within and between GPi and STN in untreated and treated patients with Parkinson's disease. Off levodopa LPs in both nuclei were dominated by low-frequency activity with coherence between the two signals at $\sim 6$ and $20 \mathrm{~Hz}$. Conversely, after treatment with levodopa doses sufficient to improve parkinsonism, this low-frequency synchronization was diminished and replaced by synchronization at $\sim 70 \mathrm{~Hz}$. Power changes were in the same direction as changes in coherence so that the latter were not attributable to alterations in the nonlinear components of the signals. These dramatic effects of levodopa on pallidal-STN oscillations may have been exerted through the projection of the substantia nigra pars compacta to the putamen and thence to STN or directly via a dopaminergic substantia nigra pars compactaSTN projection (Parent and Hazrati, 1995). In line with the latter, the overall electrophysiological activity of the STN is known to increase after the direct iontophoretic application of dopamine in the rat (Cambell et al., 1985).

\section{Origin of low- and high-frequency activities}

Macroelectrode sites were deemed to be within GPi and STN on the basis of their stereotactic coordinates, postoperative MRI, and the antiparkinsonian effects of high-frequency stimulation at each site. In addition, the phase reversal of the cumulant density estimates indicated that the activities recorded by contacts GPi12 and STN12 were generated locally rather than picked up through volume conduction from more distant sources.

The networks subserving synchronized oscillations at above 15 $\mathrm{Hz}$, which represent the bulk of the activity recorded here, are obscure. To date, there have been no reports of such oscillatory activity in cross-correlations of single units within or between GPi and STN. Nevertheless, recent investigations of organotypic cocultures suggest that the STN-pallidal network can support rhythmic activity, albeit at frequencies under $4 \mathrm{~Hz}$ (Plenz and Kital, 1999). Cortical (Magill et al., 2000; Nambu et al., 2000) and subcortical influences, such as those from the striatum (Ryan and Clark, 1992), thalamus (Bevan et al., 1995; Smith et al., 1998), and pedunculopontine nucleus (Inglis and Winn, 1995), may further modify this innate rhythmicity.

\section{Phase relationships between GPi and STN}

Phase relationships between GPi and STN are open to several interpretations. However, we would choose to view them in the light of current models of the basal ganglia (Albin et al., 1989; Alexander and Crutcher, 1990; Parent and Hazrati, 1995; Chesselet and Delfs, 1996; Smith et al., 1998). Off levodopa, activity in GPi led that in STN at $\sim 20 \mathrm{~Hz}$. This would be consistent with the common driving of STN and GPi by globus pallidus externa (GPe) (with conduction and neuronal delays to STN being longer than to GPi). Also off levodopa, activity in STN at $\sim 6 \mathrm{~Hz}$ led that in GPi and might contribute to the postulated synchronization of output from GPi at low frequency in untreated Parkinson's disease (Brown and Marsden, 1998).

Dopaminergic stimulation dramatically changed the pattern of functional connectivity. Oscillatory interactions between STN and GPi were dominated by activity at $\sim 70 \mathrm{~Hz}$ in which STN led $\mathrm{GPi}$, whereas those below $30 \mathrm{~Hz}$ were greatly diminished. This would be consistent with the driving of GPi by STN at high frequency when on treatment, either through direct STN-GPi projections or via STN-GPe-GPi pathways.

The above may well not be the only interactions between GPi and STN; some may involve asynchronous and nonoscillatory activity and escape detection through frequency analysis of LPs. Nevertheless, the connections identified above are likely to be quantitatively important because the postsynaptic effects of synchronized inputs are greater than those of asynchronous ones.

\section{Effects of pathological synchronization below $30 \mathrm{~Hz}$}

In the presence of deficient dopaminergic input, as in untreated Parkinson's disease, subthalamo-pallidal networks seem to be favored that oscillate at frequencies below $30 \mathrm{~Hz}$. There appear to be two main effects. First, there are oscillations at $4-10 \mathrm{~Hz}$ in 

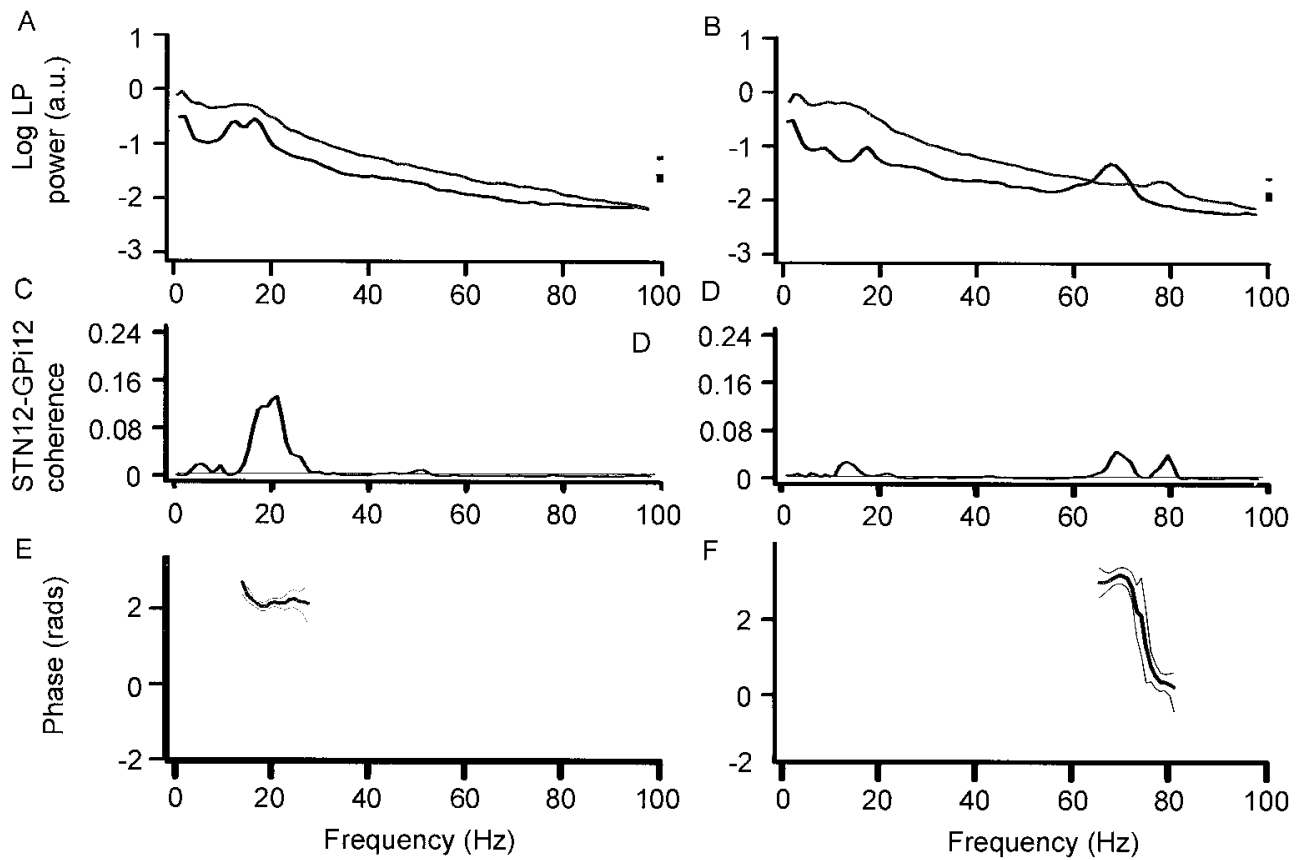

Figure 5. Autospectra of LP power ( $A$, $B)$, coherence spectra between STN12 and GPi12 $(C, D)$, and respective phase spectra $(E, F)$ after withdrawal $(A, C, E)$ or reinstitution $(B, D, F)$ of levodopa. Data pooled from all records in which the contralateral wrist was tonically extended in four patients with Parkinson's disease (over 6 experimental sessions). Off medication, there is coherence between STN12 and GPi12 at $\sim 20 \mathrm{~Hz}$. The temporal difference between STN

$\mathrm{F}$

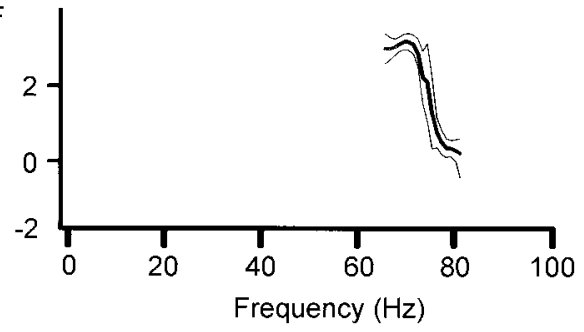
and GPi was indeterminate, with the best-fit line accounting for only $17 \%$ of the variance. The low-frequency activity is reduced on treatment when a peak appears at $\sim 70 \mathrm{~Hz}$ in the autospectrum of STN12 $(B)$ and coherence spectrum $(D)$. Regression analysis of phase suggested that STN led GPi by $47 \pm 9 \mathrm{msec}$ from 69.3 to $82.0 \mathrm{~Hz}\left(r^{2}=0.910 ; p<\right.$ $0.0001)$.

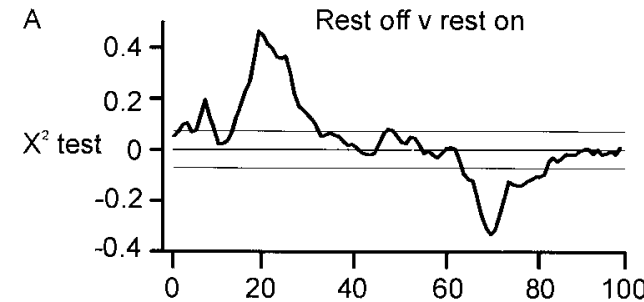

C

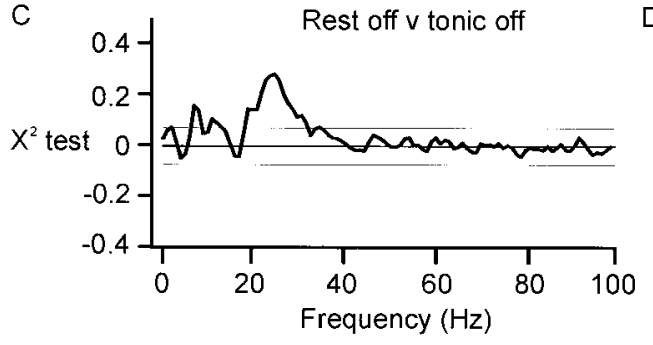

B

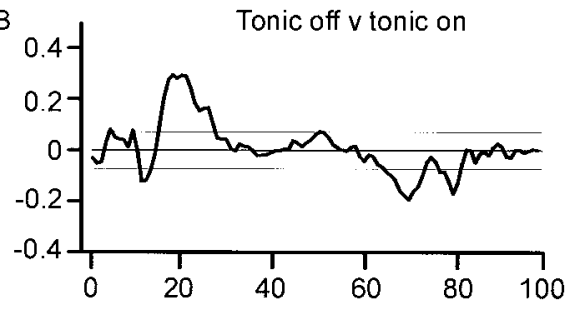

D

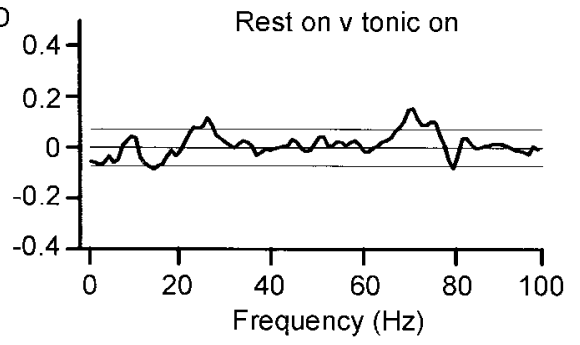

Figure 6. $A$, Comparison of pooled coherence at rest off and on levodopa. $B$, Comparison of pooled coherence during tonic wrist extension off and on levodopa. In both $A$ and $B$, coherence was greater at $\sim 20$ and $70 \mathrm{~Hz}$ off and on levodopa, respectively. $C$, Comparison of pooled coherence at rest and during tonic wrist extension when off levodopa. Coherence was greater at rest at $\sim 6$ and $20 \mathrm{~Hz}$. D, Comparison of pooled coherence at rest and during tonic wrist extension after treatment with levodopa. Coherence was greater at rest at $\sim 70 \mathrm{~Hz}$. which STN leads GPi. These oscillations may be related to tremor as reported in microelectrode single-unit studies in monkeys (Bergman et al., 1994, 1998) and parkinsonian patients (Hutchison et al., 1997b; Taha et al., 1997; Magnin et al., 2000). Certainly, in one of our cases with a florid rest tremor off medication, there was coherence between the STN LP and EMG at tremor frequency. The second oscillatory activity occurred in the $\beta$ range, with a peak centered at $\sim 20 \mathrm{~Hz}$. Here GPi phase led STN. Both activities may disrupt normal motor function (Wichmann and DeLong, 1996). Artificial driving of the pallidum in the cat (Hassler and Dieckmann, 1967; Dieckmann, 1968) or of STN in humans (Demeret et al., 1999) at low frequency brings on or exacerbates parkinsonism, suggesting that the spontaneous synchronization at low frequency may contribute to the abnormal pattern of movement in Parkinson's disease (Brown and Marsden, 1998). This hypothesis deserves further investigation, because it may help explain some of the paradoxical effects of functional neurosurgery (see below).

\section{Effects of synchronization at $\sim 70 \mathrm{~Hz}$}

Our results imply that elements within the pallidum and STN form a functional network that ordinarily, in the presence of a normal dopaminergic drive, resonates at $\sim 70 \mathrm{~Hz}$. That such a rhythm is important for the optimal organization of voluntary movement is strongly suggested by the antiparkinsonian effects of stimulation of the same nuclei at frequencies likely to cause resonance within this network, at or near the base frequency of $70 \mathrm{~Hz}$ or its second harmonic (Siegfried and Lippitz, 1994; Limousin et al., 1995; Starr et al., 1998). However, the oscillations occurring after the reinstitution of dopaminergic stimulation are unlikely to be directly related to the execution of voluntary movement because they occur at rest as well as during motor activity. As such, they could be related to attentional processes operating in the executive domain, acting through the thalamus to favor cortico-cortical interactions in the gamma band (Hassler, 1980; Brown and Marsden, 1998). In support of this is the disappearance of the $70 \mathrm{~Hz}$ activity with drowsiness in one patient, although one might have expected the 
same activity to be increased rather than reduced during tonic voluntary contraction. Alternatively, the $70 \mathrm{~Hz}$ activity could act as a carrier rhythm for motor commands. The narrow band nature of the high-frequency activity evident in individual power and coherence spectra would be particularly suited to a carrier function.

\section{Implications for functional neurosurgery}

The present findings could help explain the paradoxical results of functional neurosurgery for Parkinson's disease. Hitherto, the efficacy of this treatment has been difficult to explain in terms of the known physiology of the basal ganglia. There are two surgical techniques, lesioning of GPi or STN and stimulation of the same sites at high frequency through implanted macroelectrodes (Siegfried and Lippitz, 1994; Limousin et al., 1995; Gill and Heywood, 1997; Starr et al., 1998). Focal lesions of GPi should destroy the major output of the basal ganglia to the motor cortex and abolish their contributions to normal voluntary movement. Lesions would therefore be expected to impair motor performance, but the reverse is seen in Parkinson's disease. On the other hand, the similarity between the effects of stimulation at frequencies in excess of $50 \mathrm{~Hz}$ and focal lesioning might imply that the former works through the induction of a virtual lesion by depolarization block (Limousin et al., 1995). However, human GPi neurons discharge at frequencies of $\sim 85-140 \mathrm{~Hz}$ in Parkinson's disease, suggesting that neural elements are more likely to be driven than blocked by high-frequency stimulation (Hutchison et al., 1997a; Merello et al., 1999; Magnin et al., 2000).

These paradoxical observations could be reconciled by the present findings if we are correct in hypothesizing that the low- and highfrequency modes of the subthalamic-pallidal circuit impair and promote motor function, respectively. In this case, the low-frequency activity could be blocked with beneficial effect by either exogenous dopaminergic stimulation or the focal destruction of GPi or STN. At the same time, therapeutic stimulation of either nucleus at high frequency might artificially drive a circuit that normally requires dopaminergic stimulation to resonate in its optimal mode.

\section{REFERENCES}

Albin RL, Young AB, Penney JB (1989) The functional anatomy of basal ganglia disorders. Trends Neurosci 12:366-376.

Alexander GE, Crutcher ME (1990) Functional architecture of the basal ganglia circuits: neural substrates of parallel processing. Trends Neurosci 13:266-271.

Amjad AM, Halliday DM, Rosenberg JR, Conway BA (1997) An extended difference of coherence test for comparing and combining several independent coherence estimates: theory and application to the study of motor units and physiological tremor. J Neurosci Methods 73:69-79.

Bejjani B, Damier P, Arnulf I, Bonnet AM, Vidailhet M, Dormont D, Pidoux B, Cornu P, Marsault C, Agid Y (1997) Pallidal stimulation for Parkinson's disease: two targets? Neurology 49:1564-1569.

Bergman H, Wichmann T, Karmon B, DeLong MR (1994) The primate subthalamic nucleus. II. Neuronal activity in the MPTP model of parkinsonism. J Neurophysiol 72:507-520.

Bergman H, Feingold A, Nini A, Raz A, Slovin H, Abeles M, Vaadia E (1998) Physiological aspects of information processing in the basal ganglia of normal and parkinsonian primates. Trends Neurosci 21:32-38.

Bevan MD, Francis CF, Bolam JP (1995) The glutamate-enriched cortical and thalamic input to neurons in the subthalamic nucleus of the rat. J Comp Neurol 361:491-511.

Brown P, Marsden CD (1998) What do the basal ganglia do? Lancet 351:1801-1804.

Cambell GA, Eckardt MJ, Weight FF (1985) Dopaminergic mechanisms in subthalamic nucleus of rat: analysis using horseradish peroxidase and microiontophoresis. Brain Res 333:261-270.

Chesselet MF, Delfs JM (1996) Basal ganglia and movement disorders: an update. Trends Neurosci 18:417-422.

Creutzfeldt OD, Watanabe S, Lux HD (1966) Relations between EEG phenomena and potentials of single cortical cells. I. Evoked responses after thalamic and epicortical stimulation. Electroencephalogr Clin Neurophysiol 20:1-18.
Demeret S, Bejjani B-P, Arnulf I, Damier P, Gervais D, Houeto JL, Pridoux B, Agid Y (1999) Low frequency subthalamic stimulation worsens parkinsonian symptoms. Neurology [Suppl 2] 52:A406.

Dieckmann G (1968) Cortical synchronised and desynchronised responses evoked by stimulation of the putamen in cats. J Neurol Sci 7:385-391.

Filion M, Tremblay L (1991) Abnormal spontaneous activity of globus pallidus neurons in monkeys with MPTP-induced parkinsonism. Brain Res 547:142-151.

Frost JD (1968) EEG-intracellular potential relationships in isolated cerebral cortex. Electroencephalogr Clin Neurophysiol 24:434-443.

Gill SS, Heywood P (1997) Bilateral dorsolateral subthalamotomy for advanced Parkinson's disease. Lancet 350:1224.

Halliday DM, Rosenberg JR, Amjad AM, Breeze P, Conway BA, Farmer SF (1995) A framework for the analysis of mixed time series/point process data: theory and application to the study of physiological tremor, single motor unit discharges and electromyograms. Prog Biophys Mol Biol 64:237-278.

Hassler R (1980) Brain mechanisms of intention and attention with introductory remarks on other volitional processes. Prog Brain Res 54:585-614.

Hassler R, Dieckmann G (1967) Arrest reaction, delayed inhibition and unusual gaze behaviour resulting from stimulation of the putamen in awake unrestrained cats. Brain Res 5:504-508.

Hutchison WD, Levy R, Dostrovsky JO, Lozano AM, Lang AE (1997a) Effects of apomorphine on globus pallidus neurons in parkinsonian patients. Ann Neurol 42:767-775.

Hutchison WD, Lozano AM, Tasker AE, Dostrovsky JO (1997b) Identification and characterization of neurons with tremor-frequency activity in human globus pallidus. Exp Brain Res 113:557-563.

Hutchison WD, Allan RJ, Opitz H, Levy R, Dostrovsky JO, Lang AE, Lozano AM (1998) Neurophysiological identification of the subthalamic nucleus in surgery for Parkinson's disease. Ann Neurol 44:622-628.

Inglis WL, Winn P (1995) The pedunculopotine tegmental nucleus. Where the striatum meets the reticular formation. Prog Neurobiol 47:1-29.

Krack P, Pollak P, Limousin P, Hoffmann D, Benazzouz A, Le Bas JF, Koudsie A, Benabid AL (1998) Opposite motor effects of pallidal stimulation in Parkinson's disease. Ann Neurol 43:180-192.

Limousin P, Pollak P, Benazzouz A, Hoffmann D, Le Bas JF, Broussolle E, Perret JE, Benabid AL (1995) Effect on parkinsonian signs and symptoms of bilateral subthalamic nucleus stimulation. Lancet 345:91-95.

Magill PJ, Bolam JP, Bevan MD (2000) Relationship of activity in the subthalamic nucleus-globus pallidus network to cortical electroencephalogram. J Neurosci 20:820-833.

Magnin M, Morel A, Jeanmond D (2000) Single unit analysis of the pallidum, thalamus and subthalamic nucleus in parkinsonian patients. Neuroscience 96:549-564.

Merello M, Balej J, Delfino M, Cammarota A, Betti O, Leiguarda R (1999) Apomorphine induces changes in GPi spontaneous outflow in patients with Parkinson's disease. Mov Disord 14:45-49.

Mima T, Hallett M (1999) Corticomuscular coherence: a review. J Clin Neurophysiol 16:501-511.

Nambu A, Tokuno H, Hamada I, Kita H, Imanishi M, Akazawa T, Ikeuchi Y, Hasegawa N (2000) Excitatory cortical inputs to pallidal neurons via the subthalamic nucleus in the monkey. J Neurophysiol 84:289-300.

Nini A, Feingold A, Slovin H, Bergman H (1995) Neurons in the globus pallidus do not show correlated activity in the normal monkey, but phaselocked oscillations appear in the MPTP model of parkinsonism. J Neurophysiol 74:1800-1805.

Parent A, Hazrati L-N (1995) Functional anatomy of the basal ganglia. II. The place of the subthalamic nucleus and external pallidum in basal ganglia circuitry. Brain Res Rev 20:128-154

Plenz D, Kital ST (1999) A basal ganglia pacemaker formed by the subthalamic nucleus and external globus pallidus. Nature 400:677-682.

Ryan LJ, Clark KB (1992) Alteration of neuronal responses in the subthalamic nucleus following globus pallidus and neostriatal lesions in rats. Brain Res Bull 29:319-327.

Scatton B, Javoy-Agid F, Rouquier L, Dubois B, Agid Y (1983) Reduction of cortical dopamine, noradrenaline, serotonin and their metabolites in Parkinson's disease. Brain Res 275:321-328.

Siegfried J, Lippitz B (1994) Bilateral chronic electrostimulation of ventroposterolateral pallidum: a new therapeutic approach for alleviating all parkinsonian symptoms. Neurosurgery 35:1126-1130.

Smith Y, Bevan MD, Shink E, Bolam JP (1998) Microcircuitry of the direct and indirect pathways of the basal ganglia. Neuroscience 86:353-387.

Starr PA, Vitek J, Bakay RAE (1998) Ablative surgery and deep brain stimulation for Parkinson's disease. Neurosurgery 43:989-1015.

Sterio D, Beric A, Dogali M, Fazzini E, Alfaro G, Devinsky O (1994) Neurophysiological properties of pallidal neurons in Parkinson's disease. Ann Neurol 35:586-591.

Taha JM, Favre J, Baumann TK, Burchiel KJ (1997) Tremor control after pallidotomy in patients with Parkinson's disease: correlation with microrecording findings. J Neurosurg 86:642-647.

Wichmann T, DeLong MR (1996) Functional and pathophysiological models of the basal ganglia. Curr Opin Neurobiol 6:751-758. 